\title{
Laser characterization with advanced digital signal processing
}

Piels, Molly; Tafur Monroy, Idelfonso ; Zibar, Darko

Published in:

Proceedings of the SPIE

Link to article, DOI:

$10.1117 / 12.2078335$

Publication date:

2015

Document Version

Publisher's PDF, also known as Version of record

Link back to DTU Orbit

Citation (APA):

Piels, M., Tafur Monroy, I., \& Zibar, D. (2015). Laser characterization with advanced digital signal processing. In Proceedings of the SPIE (Vol. 9388). [938809] SPIE - International Society for Optical Engineering. Proceedings of SPIE - The International Society for Optical Engineering https://doi.org/10.1117/12.2078335

\section{General rights}

Copyright and moral rights for the publications made accessible in the public portal are retained by the authors and/or other copyright owners and it is a condition of accessing publications that users recognise and abide by the legal requirements associated with these rights.

- Users may download and print one copy of any publication from the public portal for the purpose of private study or research.

- You may not further distribute the material or use it for any profit-making activity or commercial gain

- You may freely distribute the URL identifying the publication in the public portal 


\title{
Laser characterization with advanced digital signal processing
}

Molly Piels, Idelfonso Tafur Monroy, and Darko Zibar

DTU Fotonik, Ørsteds Plads, Building 343, 2800 Kgs Lyngby, Denmark

\begin{abstract}
The use of machine learning techniques to characterize lasers with low output power is reviewed. Optimized phase tracking algorithms that can produce accurate noise spectra are discussed, and a method for inferring the amplitude noise spectrum and rate equation model of the laser under test is presented.
\end{abstract}

Keywords: Nanocavity devices, optical interconnects, digital signal processing

\section{INTRODUCTION}

The relentless growth of datacenter traffic is driving research toward lasers and detectors with lower power consumption. For low energy (10s of fJ/bit) links relying on directly-modulated laser sources, nanocavity lasers are currently considered the most promising technology, though state-of-the-art devices are not currently efficient enough for optical interconnects to be competitive with electrical ones ${ }^{1}$. Accurate component characterization is necessary in order to improve the performance of these devices as well as to evaluate their potential use in a digital link. For lasers, phase noise spectra and relative intensity noise (RIN) spectra both yield useful information about the internal device dynamics $^{2}$, and this noise can in some cases limit link performance. Unfortunately, it is often very difficult to characterize low-power-consumption lasers because the output power can be on the order of nano- or microwatts. Here, we discuss our recent research employing a coherent receiver in conjunction with Bayesian filtering to completely characterize lasers with low output power. Optimized phase and amplitude tracking algorithms can be used to generate accurate noise spectra. Traditionally, rate equation coefficients have been determined by fitting curves to these spectra ${ }^{3}$, however, we will show that rate equation models can also be constructed using a modified version of the expectation maximization algorithm, which produces more accurate results.

\section{SNR CONSIDERATIONS IN COMMUNICATION AND CHARACTERIZATION}

Both the importance and difficulty of measuring nanocavity laser noise spectra can be well-understood with simple signal-to-noise ratio (SNR) analysis of proposed digital links and measurement setups. Nanocavity lasers are currently being researched for use in applications with stringent (fJ/bit) energy consumption limits ${ }^{1,4}$; in particular, on-chip and off-chip optical interconnects. These need to operate at least at $10 \mathrm{~Gb} / \mathrm{s}$ regardless of the specific application area, while off-chip clock rates exceeding $50 \mathrm{~Gb} / \mathrm{s}$ are expected by $2022^{1}$. For obvious reasons, forward error correction (FEC) is not going to be used in these applications, and so bit error rates (BERs) on the order of $10^{-12}$ are necessary. The maximum allowable RIN level can be determined by assuming the signal is on-off keyed (OOK) and dominated by RIN from the transmitter. Assuming the RIN has a flat spectrum over the modulation bandwidth, this means that for a

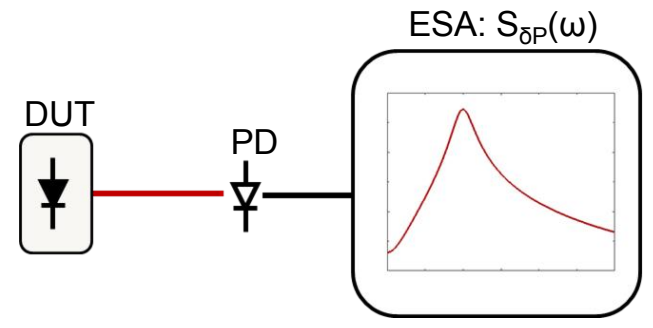

(a)

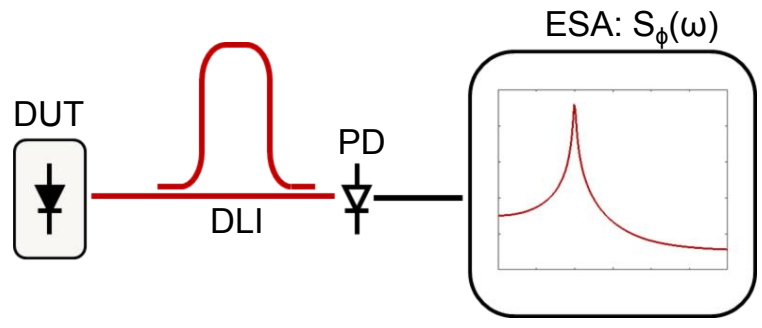

(b)

Figure 1. Analog measurement setups for characterizing laser (a) relative intensity noise spectrum $\left(\mathrm{S}_{\delta \mathrm{P}}(\omega)\right)$ and (b) FM noise $(\mathrm{S} \varphi(\omega))$. DUT: device under test; PD: (fast) photodiode; ESA: electrical spectrum analyzer; DLI: delay-line interferometer.

Optical Metro Networks and Short-Haul Systems VII, edited by Atul K. Srivastava, Benjamin B. Dingel, Achyut K. Dutta, Proc. of SPIE Vol. 9388, 938809 · @ 2015 SPIE · CCC code: 0277-786X/15/\$18 · doi: 10.1117/12.2078335 
$10 \mathrm{~Gb} / \mathrm{s}$ link, the RIN would need to be below $-120 \mathrm{dBc} / \mathrm{Hz}$, while for a $50 \mathrm{~Gb} / \mathrm{s}$ link, it would need to be less than $-127 \mathrm{dBc} / \mathrm{Hz}^{5}$. These specifications are easily met by most lasers intended for communication applications, but are actually quite close to the theoretical (quantum) limit for typical nanolaser output powers. Thus it is necessary to measure the RIN in order to evaluate whether or not a nanocavity laser can be used in an optical interconnect.

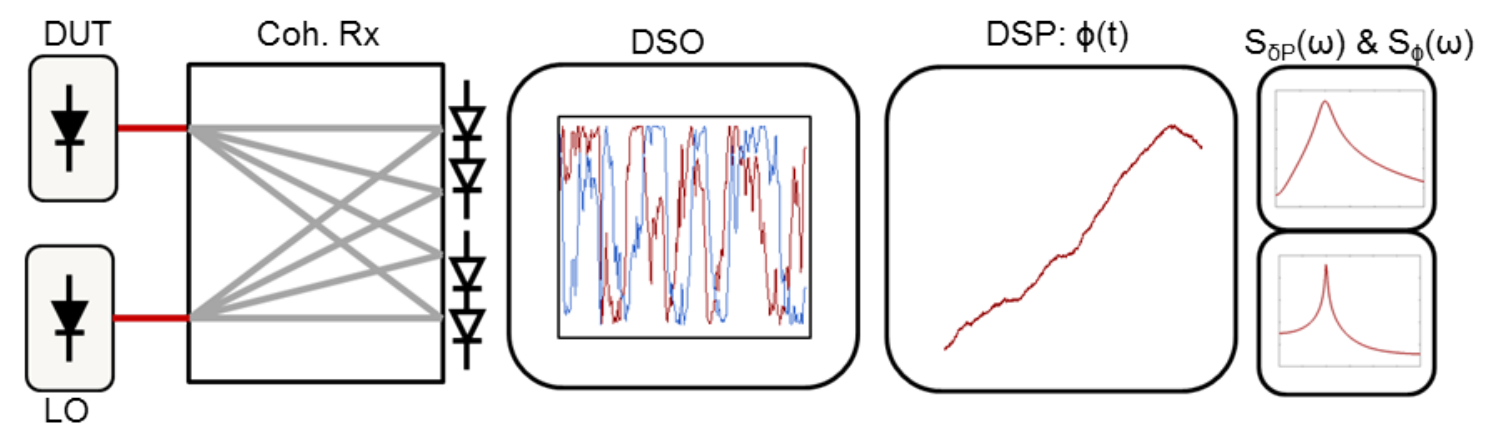

Figure 2. Coherent receiver and DSP-based measurement setup for characterizing laser RIN and FM noise spectra. LO: local oscillator; DSO: sampling oscilloscope; DSP: digital signal processing.

In addition to being an important figure of merit, characterizing the RIN of a laser with low output power is a measurement challenge. A typical RIN measurement setup is shown in Figure 1(a). The laser output is detected by a photodiode and an electrical spectrum analyzer (ESA) is used to produce the spectrum. For a device with $1 \mu \mathrm{W}$ $(-30 \mathrm{dBm})$ of optical output power, the electrical output power at DC assuming an ideal photodiode near $1550 \mathrm{~nm}$ in a 50 $\Omega$ system is $-71 \mathrm{dBm}$. In this case, problematic RIN would have a spectral density of $-191 \mathrm{dBm} / \mathrm{Hz}$ at most. This is near the shot noise limit of an ideal (noiseless electronics) system, and at least $30 \mathrm{~dB}$ below the noise floor of most commercially available ESAs. Even for a device with $100 \mu \mathrm{W}$ output power, problematic RIN would still be near the ESA noise floor. Though coherent detection (adding a local oscillator laser to the setup in Figure 1(a)) would improve the sensitivity of the measurement, due to the resulting increase in shot noise, the RIN would remain very close to, if not below, the measurement system noise floor. Thus, for lasers with very low output powers, new methods are necessary in order to determine the RIN level.

One way to estimate the RIN level is to measure the frequency (FM) noise spectrum and infer RIN from it. Measuring the FM noise spectral density does not require as high measurement sensitivity as measuring the RIN level, and the two are closely related. Theoretically, knowledge of the Schawlow-Townes linewidth and modulation response should be sufficient to determine the RIN level ${ }^{3,6}$. A traditional analog RF setup is shown in Figure 1(b). An interferometer is used to convert frequency fluctuations into amplitude fluctuations. For a laser with a $100 \mathrm{MHz}$ Lorentzian linewidth and an interferometer with $10 \mathrm{GHz}$ free spectral range, the FM noise power spectral density would be around $-100 \mathrm{dBc} / \mathrm{Hz}$. This would still constitute a measurement challenge, but less so than measuring the RIN directly. As with RIN measurements, coherent detection can be used to further increase the sensitivity. This is best accomplished by replacing the delay line interferometer with a digital coherent receiver, as shown in Figure 2. An added benefit of using a coherent receiver with balanced photodetection is that the common-mode noise (e.g. from the LO RIN) will cancel. Even with this improved measurement system sensitivity, the measured FM noise spectrum can differ substantially from the true FM noise spectrum. The remainder of this paper deals with machine learning techniques for extracting the phase from the time-domain data using this measurement setup and then relating the FM noise spectrum to the RIN.

\section{LASERS IN BAYESIAN FILTERING FRAMEWORK}

Most algorithms for Bayesian estimation require a state-space representation of the system being analyzed. The goal is to describe the system using a state vector, $\mathbf{x}_{\mathbf{n}}$, and model its evolution using the process equation $f$ :

$$
\mathbf{x}_{\mathbf{n}}=f\left(\mathbf{x}_{\mathbf{n}-1}, \mathbf{w}_{\mathbf{n}-1}\right)
$$


where $\mathbf{w}_{\mathbf{n}}$ is a noise source. Tracking is based on available data, $\mathbf{y}_{\mathbf{n}}$, which is related to the internal state of the system via the measurement equation $h_{n}$ :

$$
\mathbf{y}_{\mathbf{n}}=h_{n}\left(\mathbf{x}_{\mathbf{n}}, \mathbf{v}_{\mathbf{n}}\right)
$$

where $\mathbf{v}_{\mathbf{n}}$ is the noise of the measurement process. For a semiconductor laser, an expression for the function $\mathrm{f}$ can be derived from the laser rate equations ${ }^{8}$ :

$$
\begin{aligned}
& \frac{d \varphi}{d t}=\alpha G_{N} N+F_{\varphi} \\
& \frac{d \rho}{d t}=-\Gamma_{I} \rho+G_{N} I_{0} N+F_{\rho} \\
& \frac{d N}{d t}=G \rho-\Gamma_{N} N+F_{n}
\end{aligned}
$$

where $\varphi$ is the phase, $\rho$ is the output intensity perturbation, $\mathrm{N}$ is the excess carrier concentration, and $\alpha, G_{N}, \Gamma_{I}, \Gamma_{N}, I_{0}$ and $G$ are laser-related parameters: the linewidth enhancement factor, differential gain, intensity damping factor, carrier damping factor, steady-state output field intensity, and gain, respectively. The $F_{x}$ terms are Langevin noise forcing terms, which become $\mathbf{w}_{\mathbf{n}}$ in Equation 1. These terms are typically assumed to be zero-mean Gaussian random variables, with variances related to the physical properties of the laser. The rate equations can be translated into discrete form by defining the state and process noise vectors

$$
\mathbf{x} \equiv\left[\begin{array}{c}
f \\
\varphi \\
\rho \\
N
\end{array}\right], \text { and } \mathbf{w} \equiv\left[\begin{array}{c}
F_{f} \\
F_{\varphi} \\
F_{\rho} \\
F_{N}
\end{array}\right]
$$

where an additional state variable, $\mathrm{f}$, has been added in order to permit tracking of $1 / \mathrm{f}$ noise, and using the EulerMaruyama method":

$$
\left[\begin{array}{c}
f_{n} \\
\varphi_{n} \\
\rho_{n} \\
N_{n}
\end{array}\right]=\left(\mathbf{I}+\tau\left[\begin{array}{cccc}
0 & 0 & 0 & 0 \\
1 / \tau & 0 & 0 & \alpha G_{N} \\
0 & 0 & -\Gamma_{I} & G_{N} I_{0} \\
0 & 0 & -G & -\Gamma_{N}
\end{array}\right]\right)\left[\begin{array}{c}
f_{n-1} \\
\varphi_{n-1} \\
\rho_{n-1} \\
N_{n-1}
\end{array}\right]+\left[\begin{array}{c}
F_{f, n-1} \\
F_{\varphi, n-1} \\
F_{\rho, n-1} \\
F_{N, n-1}
\end{array}\right]
$$

where $\tau$ is the sampling period and $\mathbf{I}$ is the $4 \times 4$ identity matrix. This can be rewritten more compactly as

$$
\mathbf{x}_{\mathbf{n}}=\mathbf{A} \mathbf{x}_{\mathbf{n}-\mathbf{1}}+\mathbf{w}_{\mathbf{n}-1},
$$

where $\mathbf{A}$ is the $4 \times 4$ matrix defined in Equation 6, called the state transition matrix. It is worth noting that this equation is linear, as this simplifies the tracking problem. It is also worth noting that in principle, the same equation describes both the phase amplitude fluctuations of the laser output. To the extent that the coefficients of $\mathbf{A}$ and the probability density functions (PDFs) of the Langevin terms can be accurately estimated, the amplitude fluctuations can be determined. If the field of the laser is measured using a coherent receiver, as indicated in Figure 2, and the local oscillator has sufficiently narrow linewidth, the measurement equation $h_{n}$ can be expressed as

$$
\mathbf{y}_{\mathbf{n}}=\left(1+\rho_{n}\right)\left[\begin{array}{c}
\cos \left(\varphi_{n}\right) \\
\sin \left(\varphi_{n}\right)
\end{array}\right]+\mathbf{v}_{\mathbf{n}} .
$$

In this case, the vector $\mathbf{v}_{\mathbf{n}}$ with consist of two zero-mean Gaussian random variables with variances given by the signalto-noise ratio of the measurement system. It is worth noting that equation is not linear, and that if the intensity noise of the laser is below the noise floor of the measurement system, $1+\rho_{n} \approx 1$. 


\section{KALMAN AND PARTICLE FILTERS}

The machine learning approach to tracking and estimation is to reformulate Equations 1-2 in terms of probabilities. The challenge is to estimate the probability of $\mathbf{x}_{\mathbf{n}}$ given all prior measured values, or to find an expression for $p\left(\mathbf{x}_{\mathbf{n}} \mid \mathbf{y}_{1: \mathbf{n}}\right)$. The estimated value of $\mathbf{x}_{\mathbf{n}}$ at each time step is then chosen as the value which maximizes this likelihood function. It can be shown that, when both the process equation and the measurement equation are linear, and when both the process noise and the measurement noise are Gaussian, that the Kalman filtering algorithm produces the maximally likely estimate of $\mathbf{x}_{\mathbf{n}}$. The Gaussian assumption allows the process and measurement noise to be parameterized by covariance matrices. The effect of process noise on measurement data variation can then be evaluated analytically because the system is linear, which in turn enables analytical determination of the maximally likely current state from the measured data. A summary of the filtering equations is reproduced here for completeness; a full description can be found in many sources on the subject, e.g. ${ }^{10}$

$$
\begin{array}{ll}
\hat{\mathbf{x}}_{n}^{-}=\mathbf{A} \hat{\mathbf{x}}_{n-1}^{-} & \text {State estimate propagation } \\
\mathbf{P}_{\mathbf{n}}^{-}=\mathbf{A} \mathbf{P}_{\mathbf{n}-\mathbf{1}}^{-} \mathbf{A}^{\mathbf{T}}+\mathbf{Q} & \text { Error covariance propagation } \\
\mathbf{K}_{\mathbf{n}}=\mathbf{P}_{\mathbf{n}}^{-} \mathbf{H}_{\mathbf{n}}^{\mathbf{T}}\left[\mathbf{H}_{\mathbf{n}} \mathbf{P}_{\mathbf{n}}^{-} \mathbf{H}_{\mathbf{n}}^{\mathbf{T}}+\mathbf{R}\right]^{-1} & \text { Kalman gain update } \\
\hat{\mathbf{x}}_{\mathbf{n}}=\hat{\mathbf{x}}_{\mathbf{n}}^{-}+\mathbf{K}_{\mathbf{n}}\left(\mathbf{y}_{\mathbf{n}}-\mathbf{H}_{\mathbf{n}} \hat{\mathbf{x}}_{\mathbf{n}}^{-}\right) & \text {State estimate update } \\
\mathbf{P}_{\mathbf{n}}=\left(\mathbf{I}-\mathbf{K}_{\mathbf{n}} \mathbf{H}_{\mathbf{n}}\right) \mathbf{P}_{\mathbf{n}}^{-} & \text {Error covariance update }
\end{array}
$$

Here, $\mathbf{P}_{\mathbf{n}}$ is the estimated covariance matrix of the process noise, $\mathbf{Q}$ is the initial guess for the process noise covariance matrix, $\mathbf{R}$ is the measurement noise covariance matrix, and $\mathbf{H}_{\mathbf{n}}$ is the matrix form of the measurement equation. The process noise is parameterized by the covariance matrix, and its effect on the variation in the measurement can be evaluated exactly. This makes it possible to determine, at each time step, When the phase is measured using a coherent receiver, the measurement equation (Equation 8) is nonlinear. In this case, the extended Kalman filter (EKF) can be used instead, though it is no longer optimal. For the EKF, the measurement equation is linearized around the measured point:

$$
\mathbf{H}_{\mathbf{n}}=\left.\frac{d h}{d \mathbf{x}}\right|_{\mathbf{x}=\hat{\mathbf{x}}_{n \mid n-1}}=\left[\begin{array}{cccc}
0 & -\left(1+\hat{\rho}_{n \mid n-1}\right) \sin \left(\hat{\varphi}_{n \mid n-1}\right) & \cos \left(\hat{\varphi}_{n \mid n-1}\right) & 0 \\
0 & \left(1+\hat{\rho}_{n \mid n-1}\right) \cos \left(\hat{\varphi}_{n \mid n-1}\right) & \sin \left(\hat{\varphi}_{n \mid n-1}\right) & 0
\end{array}\right] .
$$

In addition to the known deviation from linear, Gaussian, behavior due to the nonlinearity of the measurement equation, real lasers display nonlinear, non-Gaussian behavior. Phase noise due to pump power fluctuations, for example, involves a non-Gaussian noise forcing term ${ }^{11}$. The particle filter is a known method for tracking nonlinear, nonGaussian systems ${ }^{10,12}$. Instead of parameterizing the process noise PDF, it is approximated as a weighted sum of Gaussian random variables (particles). At each step, the function $p\left(\mathbf{x}_{\mathbf{n}} \mid \mathbf{y}_{1: \mathbf{n}}\right)$ is evaluated numerically rather than analytically. A number of different algorithms for updating particles and weights exist; in this work, we used a generic particle filter with 100 particles and systematic resampling ${ }^{12}$.

\section{PHASE AND AMPLITUDE TRACKING USING MACHINE LEARNING}

To evaluate the accuracy of machine learning techniques in tracking phase and amplitude, we simulated typical output field profiles and calculated the mean-squared error (MSE) for both ML techniques and a reference method. Simulated laser parameters can be found in Table 1, and a graphical description of the noise spectrum is in Figure 3. The sampling frequency was assumed to be $40 \mathrm{GS} / \mathrm{s}$. The differential gain $G_{N}$ and threshold gain $G$ were both chosen to be typical values for semiconductor lasers. The steady-state output intensity was calculated using typical above-threshold equations ${ }^{6}$, and assuming the threshold carrier density was $10^{17} \mathrm{~cm}^{-3}$, that the pump power was $2.5 \mathrm{x}$ its threshold value, and that the carrier lifetime was $25 \mathrm{~ns}$. The damping coefficients were calculated using the equations in $^{8}$, assuming a 
spatial hole burning coefficient of $10^{-9}$. The corresponding relaxation resonance frequency, as indicated in Figure 3 , is $1.2 \mathrm{GHz}$, while the damping frequency is $244 \mathrm{MHz}$. The covariances of the Langevin noise forcing terms other than $F_{f}$ were calculated from the aforementioned laser parameters as well as the target equivalent Lorentzian linewidth. The frequency where the $1 / \mathrm{f}$ noise crosses the flat FM noise is determined by the relative magnitudes of the variances of $F_{f}$ and $F_{\varphi}$, and is $100 \mathrm{MHz}$.

Table 1. Parameters of the laser used to validate the proposed phase tracking techniques.

\begin{tabular}{|l|l|}
\hline Parameter & Value \\
\hline$\tau$ & $25 \mathrm{ps}$ \\
\hline$\alpha$ & 1 \\
\hline $\mathrm{G}_{\mathrm{N}}$ & $1 \cdot 10^{-6} \mathrm{~cm}^{3} / \mathrm{s}$ \\
\hline $\mathrm{G}$ & $4 \cdot 10^{10} \mathrm{~s}^{-1}$ \\
\hline $\mathrm{I}_{0}$ & $1.5 \cdot 10^{15} \mathrm{~cm}^{-3}$ \\
\hline$\Gamma_{\mathrm{I}}$ & $-1.5 \cdot 10^{6} \mathrm{~s}^{-1}$ \\
\hline$\Gamma_{\mathrm{N}}$ & $1.54 \cdot 10^{9} \mathrm{~s}^{-1}$ \\
\hline
\end{tabular}

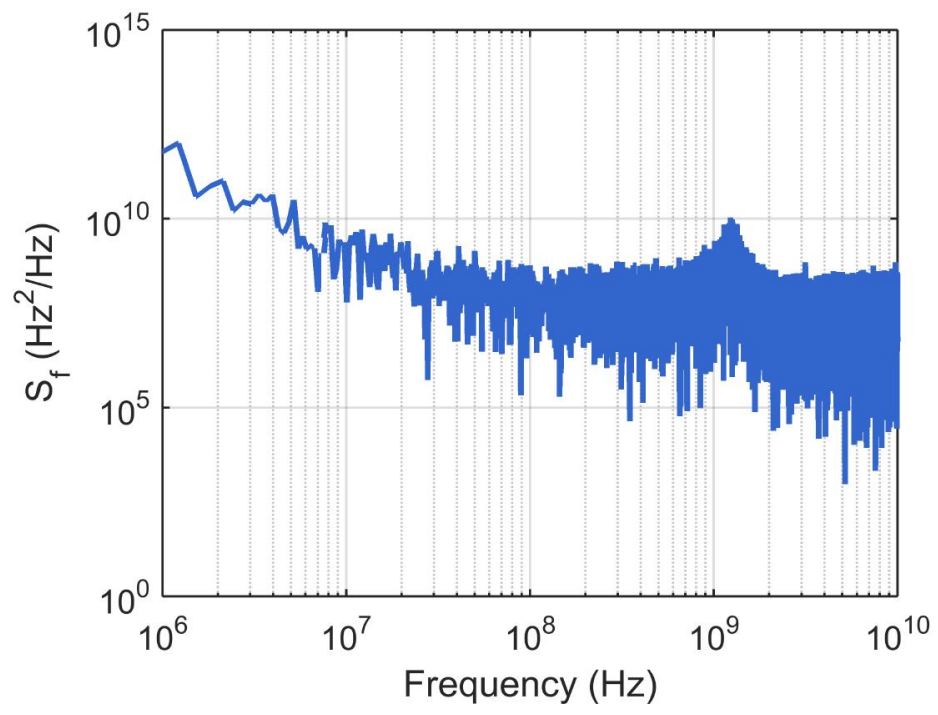

Figure 3. FM noise spectrum of the simulated laser, corresponding to the parameters in Table 1.

The phase MSE is plotted as a function of SNR in Figure 4 for the EKF, particle filter, and a reference method. The reference method consists of taking the argument of the complex electric field of the reconstructed field ${ }^{13}$. Here, we define the MSE as the error mod $2 \pi$, as these cycle slip equivalents do not affect the calculated output spectrum. We assumed additive white Gaussian noise in the optical domain was the dominant source of measurement noise. This is essentially the same as assuming that the laser output would be optically amplified; this is likely because most coherent front ends have a minimum signal power below the typical output powers of nanolasers. At low SNR, the Kalman filter performs better than the reference method. At high SNR, EKF performance becomes limited by the nonlinearity of the measurement equation. For lasers with very large linewidths, it can perform worse than the reference method, though this is not the case in this example. The particle filter is not limited by nonlinearity, and thus provides the lowest MSE regardless of the laser model used and SNR. Figure 4(b) shows the calculated FM noise spectra at $10 \mathrm{~dB}$ SNR. Using the reference method, the equivalent Lorentzian linewidth appears 9-10x larger than it is, and the relaxation resonance frequency peak is obscured by noise. In the EKF and particle filter traces, the low-frequency linewidth is estimated accurately and the relaxation resonance frequency peak is visible. This clearly demonstrates the benefits of using machine learning based techniques to calculate FM noise spectra. 

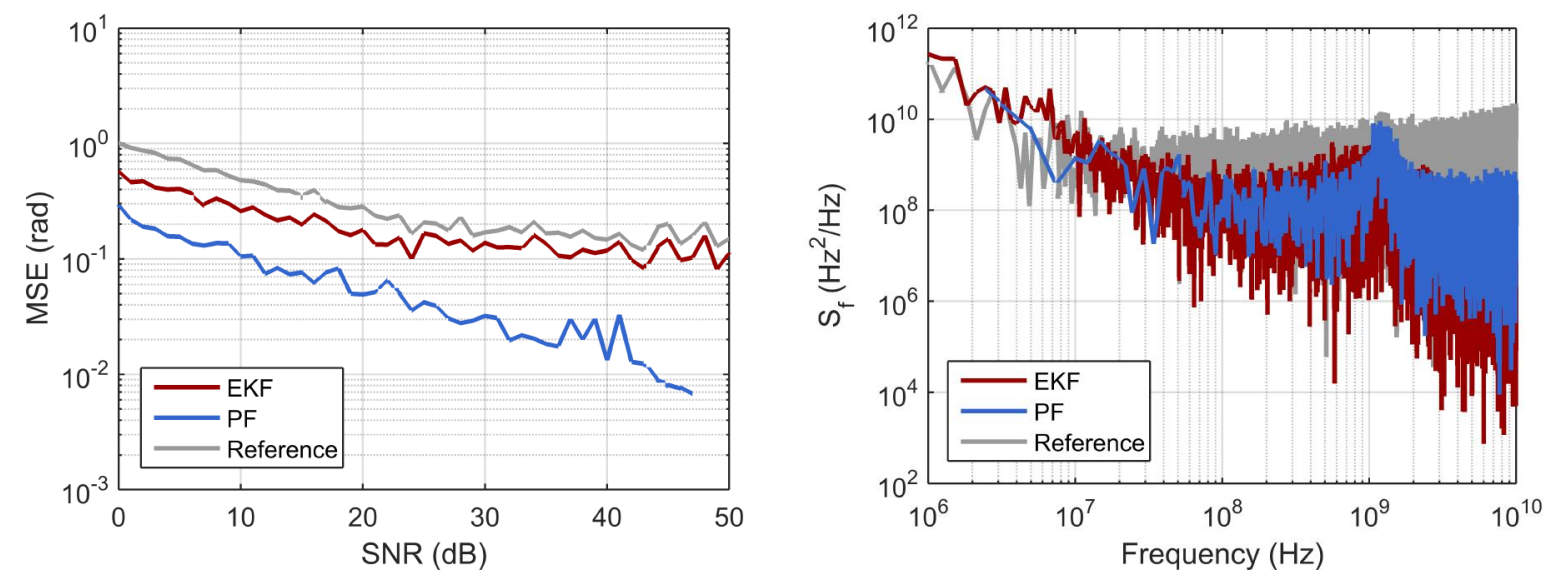

Figure 4. (a) Phase MSE as a function of measurement SNR. FM noise spectra calculated using the EKF, particle filter, and reference method at $10 \mathrm{~dB}$ SNR. Both ML techniques yield lower error than the reference method.

Figure 5 shows the MSE as a function of Lorentzian linewidth at low (5-15 dB) SNR. For narrow linewidths, the reference method is about flat, while it increases linearly for the machine learning methods. In this regime, the reference method is dominated by measurement noise, and so the phase measured is more closely related to the SNR than to the phase of the laser itself. The machine learning based methods are sensitive to the phase error variance, which is linearly proportional to the linewidth. As the linewidth exceeds $200 \mathrm{MHz}$, the performances of all three methods converge, and no method provides low MSE. In this regime, the performance of all three methods is dominated by the phase error variance. The maximum measurable linewidth can be increased by increasing the sampling rate because that will decrease the phase error variance associated with the linewidth.

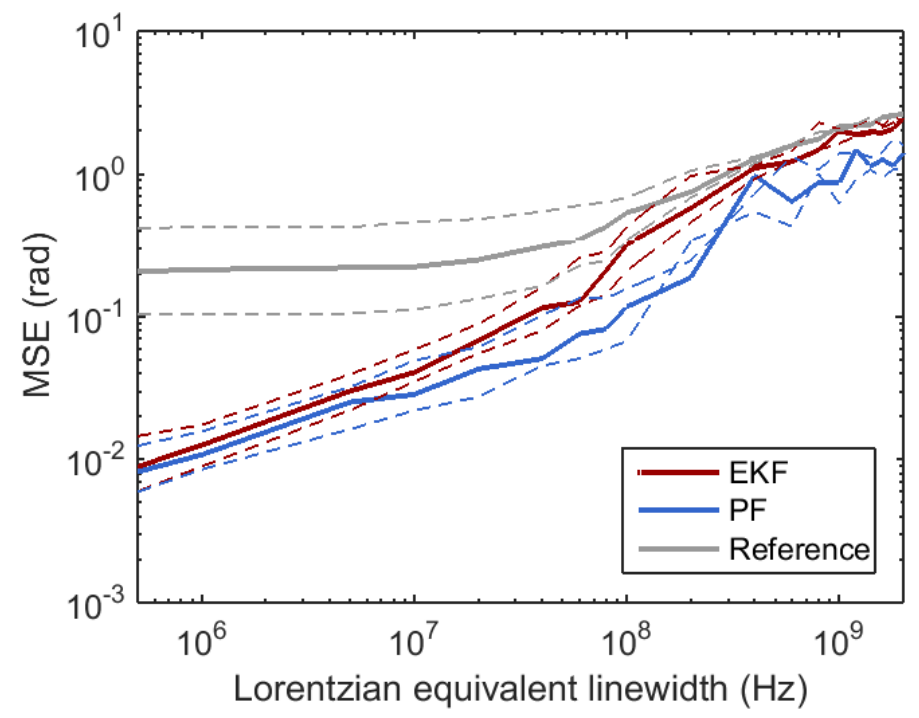

Figure 5. Phase MSE as a function of Lorentzian equivalent linewidth. Solid lines: $10 \mathrm{~dB}$ SNR. Dashed lines: 5 and $15 \mathrm{~dB}$ SNR.

\section{RIN ESTIMATION BY INFERENCE}

The relative intensity and FM noise are caused by the same physical mechanisms, and thus the RIN can be inferred from the FM noise spectrum. One common way to do this is to fit a curve parameterized by the linewidth enhancement factor 
$\alpha$, damping coefficient or damping frequency, resonance frequency, and Schawlow-Townes linewidth to the measured FM noise spectrum. The advantage of this method is that it is as robust to model parameter error as the phase tracking method used. In most parameter spaces of interest, machine learning based phase tracking methods are extremely robust to this kind of error, and the FM noise spectrum appears the same even for very inaccurate laser models. The disadvantage of the method is that it can be strongly influenced by systematic error. Low-frequency $1 / \mathrm{f}$ noise, for example, can result in a poor estimate of the Schawlow-Townes linewidth, while measurement noise tends to strongly affect the best fit linewidth enhancement factor. For the laser model studied here, this method results in an estimated low-frequency RIN level that is $10 \mathrm{~dB} / \mathrm{Hz}$ higher than the true RIN level when the spectrum is fit from $100 \mathrm{MHz}$ to $10 \mathrm{GHz}$. This is too much error for most applications of interest.

A better estimate of the RIN level can be obtained using machine learning techniques. If the transition matrix in Equation 6 accurately describes the laser, then the noise covariance matrix $\mathbf{P}$ in Equations 9-13 will converge to the covariance matrix of the Langevin noise terms. This determines the RIN level. However, obtaining an accurate parameter set for the state transition matrix can be difficult for an experimental device, as these parameters are never known with absolute certainty. One approach to this type of situation is to use expectation-maximization to determine self-consistent transition and noise covariance matrices ${ }^{14}$. However, due to the relatively large number of unobserved variables in this particular problem, this can yield non-physical results. Instead, we adopt an ad hoc approach similar to expectation maximization, where only the most difficult to measure parameters, $\Gamma_{I}$ and $\Gamma_{N}$, are allowed to vary from their initial values, and these are determined by fitting the FM noise spectrum.

Figure 6 shows an illustrative example of a RIN spectrum estimated using this technique. The parameters used to generate the phase noise were the same as in Table 1, but were modified in the filtering step. As an initial guess, we set $\Gamma_{I}$ to $10^{6} \mathrm{~s}^{-1}$ and $\Gamma_{N}$ to $10^{9} \mathrm{~s}^{-1}$, while all other parameters were set to $90 \%$ of their correct values (to emulate reasonable measurement error). The measured FM noise spectrum after this first iteration is shown in Figure 6 (a), along with a fit. The associated RIN spectrum from the incorrect model and the covariance matrix of the Langevin noise terms estimated by the EKF is shown in Figure 6 (b). Though consistent with the FM noise, the estimated RIN is many orders of magnitude larger than the true RIN due to error in the system model. After the initial FM spectrum calculation, curves are fit to find more accurate values for $\Gamma_{I}$ and $\Gamma_{N}$. Leaving all other parameters at their slightly incorrect values, the trace is processed a second time with the new damping coefficients to obtain a better RIN estimate. This is shown in Figure $6(\mathrm{c})$, along with the spectrum obtained directly from the model. There is very close agreement between true and estimated RIN power levels. Additional iterations do not yield substantially improved results. Figure 6 (d) shows the error in estimated low-frequency RIN as a function of model specification error (for simplicity, all parameters are assumed to have the same initial error). The RIN can be estimated within $5 \mathrm{~dB} / \mathrm{Hz}$ as long as these parameters are known within $30 \%$ of their correct values.

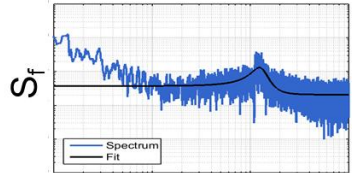

(a)

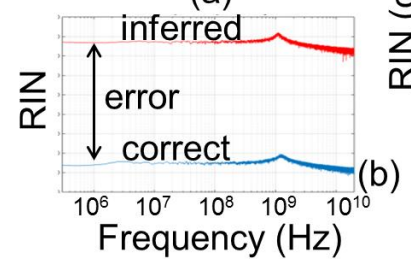

Frequency $(\mathrm{Hz})$

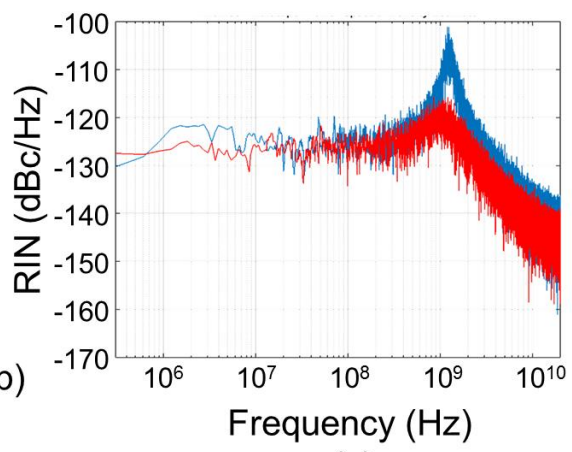

(c)

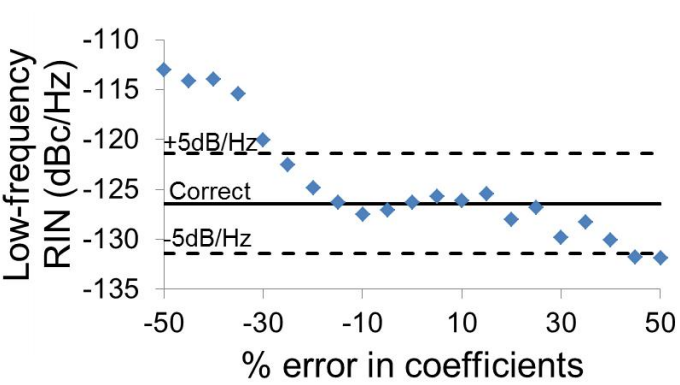

(d)

Figure 6. (a) FM noise spectrum and fit calculated using incorrect/approximate values in state transition matrix. (b) Associated estimated RIN spectrum, with correct RIN spectrum shown for comparison. (c) Correct and estimated RIN spectra after one iteration of ad hoc coefficient adjustment method. (d) Estimated low-frequency (flat) RIN level as a function of initial error in laser parameters. 


\section{REFERENCES}

[1] Miller, D., "Device Requirements for Optical Interconnects to Silicon Chips," Proc. IEEE 97(7), 1166-1185 (2009).

[2] Vahala, K., Yariv, A., "Semiclassical theory of noise in semiconductor lasers - Part II," IEEE J. Quantum Electron. 19(6), 1102-1109 (1983).

[3] Kikuchi, K., Okoshi, T., "Measurement of FM Noise, AM Noise, and Field Spectra of $1.3 \mu \mathrm{m}$ InGaAsP DFB Lasers and Determination of the Linewidth Enhancement Factor," IEEE J. Quantum Electron. QE-21(11), 18141818 (1985).

[4] De La Rue, R. M., Siyuan, Y., Lourtioz, J.-M., eds., [Compact Semiconductor Lasers], Wiley-VCH Verlag GmbH \& Co. KGaA, Weinheim, Germany (2014).

[5] Agrawal, G. P., "Optical Receivers,” [Fiber-Optic communication Systems], John Wiley \& Sons, Inc. (2002).

[6] Coldren, L. A.., Corzine, S. W., [Diode Lasers and Photonic Integrated Circuits], 1st ed., John Wiley \& Sons, Inc., New York, USA (1995).

[7] Derickson, D., [Fiber optic test and measurement], Prentice Hall, Inc., Upper Saddle River, New Jersey (1998).

[8] Henry, C., "Phase noise in semiconductor lasers," J. Light. Technol. 4(3), 298-311 (1986).

[9] Higham, D. J., "An Algorithmic Introduction to Numerical Simulation of Stochastic Differential Equations," SIAM Rev. 43(3), 525-546, Society for Industrial and Applied Mathematics (2001).

[10] Haug, A. J., "MITRE TECHNICAL REPORT A Tutorial on Bayesian Estimation and Tracking Techniques Applicable to Nonlinear and Non-Gaussian Processes" (2005).

[11] Agrawal, G. P., Roy, R., "Effect of injection-current fluctuations on the spectral linewidth of semiconductor lasers," Phys. Rev. A 37(7), 2495-2501 (1988).

[12] Arulampalam, M. S., Maskell, S., Gordon, N., Clapp, T., "A tutorial on particle filters for online nonlinear/nonGaussian Bayesian tracking,” IEEE Trans. Signal Process. 50(2), 174-188 (2002).

[13] Kikuchi, K., "Characterization of semiconductor-laser phase noise and estimation of bit-error rate performance with low-speed offline digital coherent receivers," Opt. Express 20(5), 5291-5302 (2012).

[14] Bishop, C. M., [Pattern Recognition and Machine Learning], 1st ed., Springer Science+Business, New York, USA (2006). 